\title{
Coming together in the Region to tackle COVID-19
}

\author{
Ahmed Al-Mandhari ${ }^{1}$
}

${ }^{1}$ Regional Director, World Health Organization Regional Office for the Eastern Mediterranean, Cairo, Egypt.

Citation: Al-Mandhari A. Coming together in the Region to tackle COVID-19. East Mediterr Health J. 2020;26(9):992-993 https://doi. org/10.26719/2020.26.9.992

Copyright ( ) World Health Organization (WHO) 2020. Open Access. Some rights reserved. This work is available under the CC BY-NC-SA 3.0 IGO license (https://creativecommons.org/licenses/by-nc-sa/3.o/igo)

The COVID-19 pandemic continues to pose multiple health challenges in the Eastern Mediterranean Region. Morbidity and mortality from the disease remain a serious cause for concern. As of 31 August 2020, a total of 1924511 laboratory-confirmed cases of SARS-CoV-2 virus infection had been reported in the Region, including 51019 deaths (1). Moreover, there are worrying signs that cases are now rising again in some countries after a period of decline (2) and the indirect impact of the pandemic on health care is arguably even more troubling. Access to essential health services is being compromised as scarce resources are diverted to fight the pandemic, social restriction measures such as lockdowns disrupt service provision, while fear and rumour deter people from approaching health facilities. Initial studies indicate that services such as immunization, elective surgery and chronic disease management have been severely affected (1). The long-term consequences threaten to be grave indeed.

Therefore, it is no surprise that assessing and mitigating the impacts of the pandemic are prominent on the agenda for the upcoming 67th session of the Regional Committee for the Eastern Mediterranean (3), WHO's governing body in the Region. The Committee's annual meeting is a crucial formal mechanism for ensuring the Organization's accountability to our Member States in the Region; the core of each year's session is delegates' discussion, scrutiny and approval of reports and proposals regarding technical programmes of work. No less importantly, it also provides an opportunity for informal discussions among WHO staff, Member State policy-makers and technical experts, and valued partners including WHO's sister United Nations agencies, nongovernmental organizations and other stakeholders. Through side meetings, exhibitions and social events, experiences and ideas are shared to the great mutual benefit of participants.

Unfortunately, that broader interaction is going to be diminished this year, as the Regional Committee session has itself been impacted by the pandemic. In place of the normal three to four days of face-to-face meetings, which be both imprudent and logistically challenging at present, the 67th session will be a virtual event held through an online platform. To minimize the risk of connectivity problems and accommodate different time zones, the duration will be limited to two five-hour sessions held on 12 and 13 October. The agenda has been pared down to a minimum of essential items, and some of those items will not be discussed during the Committee meeting itself, but instead circulated as documents for Member States' consideration (4,5). With time at a premium, participants will be encouraged to submit written or video statements on agenda items in advance, rather than intervening during the session, and the WHO Secretariat is holding informal consultations with Member States in advance of the meeting to help ensure that technical proposals reflect a broad consensus.

Member States will be considering some bold new initiatives. COVID-19 has highlighted concerns about the limited availability of medicines and vaccines in the Region. There have been long-standing and deep-seated issues in this regard in most countries of the Region, but the pandemic has brought them into sharp relief. Countries rely heavily on imports of medicines, so the disruption of international supply lines has led to severe shortages, making policy-makers even more anxious to ensure that adequate stocks of any eventual COVID-19 vaccine can be delivered promptly to their populations in good time. In response, WHO has developed a regional strategy to improve access to medicines and vaccines in the Eastern Mediterranean Region, 2020-2030 (6). The new strategy is based on a comprehensive analysis of gaps and challenges in the Region, and specifies comprehensive actions for both national governments and WHO's technical staff to address them. In spurring its development, the pandemic may yet prove to have a very substantial silver lining.

There is also a proposal to establish a new regional Subcommittee on Polio Eradication and Outbreaks (7). Eradication of wild poliovirus from WHO's African region was formally certified in August 2020 (8), making the Eastern Mediterranean Region the only part of the world in which it remains endemic. Indeed, there has been an alarming spike in cases recently (9). It is hoped that the new subcommittee, to be comprised of ministers of health from countries of the Region and other senior figures, can help galvanize efforts to finally end this disease. The subcommittee will also work to ensure that the experience, reach and other assets of the polio programme benefit other immunization campaigns and public health projects as well.

Current circumstances are certainly notideal but thanks to the engagement of WHO staff, our Member States and partners, we are looking forward to a constructive and productive Regional Committee session. 


\section{References}

1. The COVID-19 pandemic in the Eastern Mediterranean Region (EM/RC67/7). Technical paper to be presented to the 67th session of the WHO Regional Committee for the Eastern Mediterranean, 2020 (forthcoming).

2. Eastern Mediterranean Regional Office COVID-19 Dashboard [website]. Cairo: WHO Regional Office for the Eastern Mediterranean; 2020 (https://app.powerbi.com/view?r=eyJrIjoiN2ExNWI3ZGQtZDk3MyooYzE2LWFjYmQtNGMwZjkoOWQ1MjFhIiwidCI6ImY2MTBjMGI3LWJkMjQtNGIzOSo4MTBiLTNkYzI4MGFmYjU5MCIsImMiOjh9, accessed 19 September, 2020).

3. Regional Committee for the Eastern Mediterranean, 67th session, provisional agenda (EM/RC67/1). (forthcoming).

4. Special procedures for the virtual 67th session of the WHO Regional Committee for the Eastern Mediterranean (EM/RC67/2). Draft decision to be presented to the 67th session of the WHO Regional Committee for the Eastern Mediterranean, 2020 (forthcoming).

5. The written silence procedure (EM/RC67/3). Draft decision to be presented to the 67th session of the WHO Regional Committee for the Eastern Mediterranean, 2020 (forthcoming).

6. Regional strategy to improve access to medicines and vaccines in the Eastern Mediterranean, 2020-2030, including lessons from the COVID-19 pandemic (EM/RC67/6). Technical paper to be presented to the 67th session of the WHO Regional Committee for the Eastern Mediterranean, 2020 (forthcoming).

7. Establishment of a Regional Subcommittee for Polio Eradication and Outbreaks (EM/RC67/17). Technical paper to be presented to the 67th session of the WHO Regional Committee for the Eastern Mediterranean, 2020 (forthcoming).

8. Global polio eradication initiative applauds WHO African region for wild polio-free certification (news release, 25 August 2020). In: WHO newsroom [website]. Geneva: World Health Organization; 2020 (https://www.who.int/news-room/detail/25-08-2020-global-polio-eradication-initiative-applauds-who-african-region-for-wild-polio-free-certification, accessed 19 September 2020).

9. Progress report on eradication of poliomyelitis (EM/RC67/INF.DOC.1). Report to be presented to the 67th session of the WHO Regional Committee for the Eastern Mediterranean, 2020 (forthcoming). 